\title{
ON MINIMAX THEORY IN TWO HILBERT SPACES
}

\section{E. M. EL-KHOLY}

Faculty of Science

Department of Mathematics

Tanta University

Tanta - Egypt

\section{HANAN ALI ABDOU}

Faculty of Education

Department of Mathematics

Ain Shams University

Cairo - Egypt

(Received February 1, 1994 and in revised form April 19, 1995)

ABSTRAC'T. In this paper, we investigated the minimax of the hifunction

$$
J: H^{1}(\Omega) \times V_{2} \rightarrow R^{m} \times R^{n}
$$

such that

$$
J\left(v_{1}, v_{2}\right)=\left(\left(\frac{1}{2} a\left(v_{1}, v_{1}\right)-I\left(v_{1}\right)\right), v_{2}\right)
$$

where

a (.,.) is a finite symmetric bilinear bicontinuous, coercive form on $H^{1}(\Omega)$ and $L$ belongs to the dual of $H^{1}(\Omega)$.

In order to obtain the minimax point we use lagrangian functional.

KEY WORIS AND PHRASES. HIIlbert spaces, dual spaces, minimization of functionals, minimax point, saddle point, concave functions, convex function, bicontinuous form, coercive form.

\section{INTRODUCTION.}

The minimization of functonals defined only on one Hilbert space has been studied by Jean Cea[1], and the saddle functions has been introduced and investigated by Rockafellar [2]. Our aim, is to generalize the above work in order to obtain a general minimax of functionals defined on two Hilbert spaces. We consider the Sobolev space $H^{\prime}(\Omega)$ with inner-product,

$$
((u, v))=\int_{v}\left\{u v+\sum_{j=1}^{n}\left(D_{j} u\right)\left(D_{j} v\right)\right\} d x \text {, }
$$

as a Hilbert space.

The Green's formula will be used to obtain a minimal solution in this work

\section{PRIMILARIES}

Let $\Omega$ be a bounded open subset in $R^{n}$ and $\Gamma$ denote its boundary. The Sobolev space $H^{1}(\Omega)$ can be defined as follows, [1]:

$$
H^{1}(\Omega)=\left\{v: v \in L^{2}(\Omega), \partial v / \partial x_{j} \in L^{2}(\Omega), J=1,2, \ldots, n\right\}
$$

where $D_{j} v=\partial v / \partial x_{j}$ are taken in the sense of distributions i.e.

$$
\left\langle D_{j}, v, \varphi\right\rangle=-\left\langle v, D_{j} \varphi\right\rangle \quad \text { for all } \varphi \in D(\Omega)
$$

are $D(\Omega)$ denotes the space of all $C^{\infty}$-functions with compact support in $\Omega$, Also $<,>$ denotes the duality between $\delta(\Omega)$ and the space of distribution $D^{\prime}(\Omega)$ on $\Omega$. The space $H^{1}(\Omega)$ is provided with the inner-product. 


$$
\begin{aligned}
((u, v))=: & (u, v)_{L^{2}(0)}+\sum_{j=1}^{n}(D, u, D, v)_{L^{2}(0)} \\
& =\int_{a}\left\{u v+\sum_{j=1}^{n}(D, u)(D, v)\right\} d x .
\end{aligned}
$$

The vector $\varphi \in V, \varphi \neq 0$ is called a direction in $V$.

If $\Gamma$ is " regular" ( for instance, $\Gamma$ is a $C^{1}$ ( or $C^{\infty}$ )-manifold of dimension $n-1$ ) then the linear mapping $v \rightarrow v$ of $C^{\prime}(\bar{\Omega}) \rightarrow C^{\prime}(I)$ (resp. of $C^{\infty}(\bar{\Omega}) \rightarrow C^{\infty}(I)$ extends to a continuous linear map of $H^{1}(\Omega)$ into $L^{2}(\Gamma)$ denoted by $\gamma$ and for $v \in H^{1}(\Omega), \gamma(v)$ is called the trace of $v$ on $r$.

A linear tranformation $L: v \rightarrow L(v)$ is continuous if there exists a constant $N$ such that

$$
L,(v) \leq N\|v\|_{V} \quad \text { for } \quad v \in V \text {. }
$$

A bilinear function $a(u, v): V \times V \rightarrow R^{m}$, is bicontinuous if there exists a constant $M>0$ such that

$$
a(u, v) \leq M\left\|_{u}\right\|_{v}\|v\|_{v} \quad \text { for all } u, v \in V .
$$

A biliniear function $a(u, v)$ is $V$-coercive if there exists a constant $\alpha>0$ such that

$$
a(u, v) \geq \alpha\|v\|_{v}^{2} \quad \text { for all } v \in V \text {. }
$$

Let $E$ be a vector space, a cone with vertex at 0 in $E$ is a subset $\Lambda$ of $E$ such that, if $\lambda$ belongs to $\Lambda$ and if $\alpha$ belongs to $R$ with $\alpha \geq 0$ then $\alpha \lambda$ also belongs to $\Lambda$.

In the following we have collected the L,emmas and Theorems we needed to obtain our main results. It should be noted that these are based on [1] except corollary (4) on [2].

1. LEMMA. The following equality

$$
\sum_{j=1}^{n}(D, u, D, v)_{l^{2}(0)}=-\int_{0}(\Delta u) v d x+\int_{\Gamma} \frac{\partial u}{\partial \underline{n}} v d \sigma
$$

where

$$
\left(D_{1} u, D_{j} v\right)_{L^{2}(\Omega)} \text { is the inner product defined in } L^{2}(\Omega) \text { is true. }
$$

PROOF. If $u, v \in C^{1}(\Omega)$, and by using Green's formula [3], we get

$$
\int_{0}(D, u) v d x=-\int_{0} u\left(D_{,}, v\right) d x+\int_{\Gamma} u v n_{j} d o
$$

where do is the area element on $\Gamma$.

We define the operator of exterior normal derivation formally as

$$
\frac{\partial}{\partial \underline{n}}=\sum_{j=1}^{n} n_{j}(x) D_{j} \text {, }
$$

such that $\underline{n}(x)$ is the unique outer normal vector at each point $x$ on $\Gamma$, and $\left(\underline{n}_{1}(x), \ldots, \underline{n}_{n}(x)\right)$ are the direction cosines of $\underline{n}(x)$.

Next, if $u, v \in C^{2}(\bar{\Omega})$, then applying the above formula to $D_{j} u, D_{j} v$ becomes

$$
\left.\int_{0}(1)^{2} u\right) v d x=-\int_{0}(1, u)(D, v) d x+\int_{r}(1, u) v n, d o
$$

and

$$
\sum_{j=1}^{n} \int_{Q}\left(D_{j} u\right)\left(D_{j}, v\right) d x=-\sum_{j=1}^{n} \int_{Q}\left(D_{j}^{2} u\right) v d x+\sum_{j=1}^{n} \int_{r}\left(D_{j} u\right) v n_{j} d \sigma .
$$

Then

$$
\left.\sum_{j=1}^{n}\left(D_{j} u, D_{j} v\right)_{L^{2}(D)}=-\sum_{j=1}^{n} \int_{0}(J)_{j}^{2} u\right) v d x+\int_{\Gamma} \frac{\partial u}{\partial \underline{n}} v d \sigma
$$




$$
\sum_{j=1}^{n}(D, u, D, v)_{l^{2}(0)}=--\int_{0}(\Delta u) v d x+\int_{r} \frac{\partial u}{\partial \underline{n}} v d o
$$

2. LEMMA. Let $V$ be a Hilbert space, $V$ be its strong dual, and $J: V \rightarrow R$ be a functional such that

$$
J(v)=\frac{1}{2} a(v, v)-L(v)
$$

where $a($,$) is a symmetric bilinear, bicontinuous, coercive form on V$ and $L \in V$. Further, let $K$ be a closed convex subset of $V$ then for all $v \in K$ there exist $u \in K$ such that

$$
\mathbf{J}(\mathbf{u}) \leq \mathbf{J}(\mathbf{v})
$$

3. THEOREM. There exists a unique solution $u \in K$ which minimizes $J$ on $K$, and this problem is equivalent to the following variational problem: Find $u \in K$ such that $\mathbf{a}(\mathbf{u}, \mathbf{v}-\mathbf{u}) \geq \mathbf{L}(\mathbf{v}-\mathbf{u})$

4. COROLLARY. Let $C$ and $D$ be non-empty closed convex sets in $R^{m}$ and $R^{\star}$, respectively, and let $K$ be any finite continuous concave-convex function on $C \times D$.

Let $\underline{K}$ and $\bar{K}$ be the lower and upper simple extensions of $K$ to $R^{m} \times R^{n}$, respectively. Then $\underline{K}$ is lower closed, $\bar{K}$ is upper closed, and there exists a unique closed convex bifunction from $R^{m}$ to $R^{n}$ such that

The bifunctions $F$ and $F$ are expressed in terms of $K$ by

$$
\underline{\mathbf{K}}\left(\mathbf{u}, \dot{x}^{*}\right)=\left\langle\mathbf{F u}, \dot{x}^{*}\right\rangle, \quad \overline{\mathbf{K}}(\mathbf{u}, \dot{x})=<\mathbf{u}, \dot{\mathbf{F}} \dot{x}>\text {. }
$$

$$
\begin{aligned}
& (F u)(x)= \begin{cases}\sup \{\langle x, \dot{x}\rangle-K(u, \dot{x}) ; \dot{x} \in D\} & \text { if } u \in C, \\
+\infty & \text { if } u \notin C,\end{cases} \\
& \left(\vec{F}^{*}\right)\left(u^{*}\right)= \begin{cases}\inf \left\{<u, u^{*}>-K\left(u, \dot{x}^{*}\right) ; u \in C\right\} & \text { if } \dot{x} \in \mathbf{D}, \\
-\infty & \text { if } \dot{x} \notin D .\end{cases}
\end{aligned}
$$

In particular, dom $\mathrm{F}=\mathrm{C}$ and $\operatorname{dom} \mathrm{F}^{\star}=\mathrm{D}$.

5. THEOREM. (Ky Fan and Sion)

Let $\mathrm{V}$ and $\mathrm{E}$ be two Hausdorf topological vector spaces, $U$ be a convex compact subset of $V$ and $\Lambda$ be a convex compact subset of $E$. Suppose

$$
\mathcal{C}: \mathrm{U} \times \Lambda \rightarrow \mathrm{R}
$$

be a functional such that

i) for every $v \in U$ the functional

$$
\mathcal{C}(v, .): \mu \rightarrow \mathcal{C}(v, \mu)
$$

is upper-semi-continuous and concave,

ii) for every $\mu \in \Lambda$ the functional

$$
\mathcal{L}(., \mu): v \rightarrow \mathscr{L}(\mathrm{v}, \mu)
$$

is lower-semi-continuous and convex. Then there exists a saddle point $(u, \lambda) \in U \times \Lambda$ for

3. MAIN RESULTS.

6. THEOREM. Let $\Omega$ be a bounded open set in $R^{n}$ with smooth boundary $\Gamma$. Let $v_{1}$ and $V_{2}$ be two Hilbert spaces, $V_{1}=H^{\prime}(\Omega)$, and $V_{1}^{\prime}, V_{2}^{\prime}$ be two ( strongly) duals. If the bifunction 


$$
J: V_{1} \times V_{2} \rightarrow R^{m} \times R^{n}
$$

is defined by

$$
J\left(v_{1}, v_{2}\right)=\left(\left(\frac{1}{2} a\left(v_{1}, v_{1}\right)-L\left(v_{1}\right)\right), v_{2}\right)
$$

where $a($,$) is a finite symmetric bilinear, bicontinuous, coercive form on v_{1}, L \in V_{1}^{\prime}$, and $K_{1}$ is the following subset:

$$
K_{1}=\left\{v_{1} ; v_{1} \in H^{1}(\Omega),\left\|\gamma v_{1}\right\|_{L}^{2}(\Gamma) \leq 1 ; \gamma\left(v_{1}\right) \text { is a trace }\right\} .
$$

Then there exist a minimax of. .

PROOF. We first prove that $K_{1}$ is a closed convex set. To show that it is closed, we suppose $\left(v_{1}^{n}\right) \in K_{1}$ is a sequence such that $\left(v_{1}^{n}\right) \rightarrow v_{1}$ in $v_{1}$, and since

$$
\gamma: H^{1}(\Omega) \rightarrow I^{2}(\Gamma)
$$

is a continuous linear map. Then

$$
\gamma\left(v_{1}^{n}\right) \rightarrow \gamma\left(v_{1}\right) \text { in } I^{2}(\Gamma) .
$$

If $\varphi \in \mathrm{L}^{2}(\Gamma)$ is such that $\varphi>0$ on $\Gamma$, then

we choose $\|\Phi\|_{L}^{2}(\mu)$ such that

$$
\begin{aligned}
\int_{\Gamma}\left|\gamma\left(v_{1}\right) \varphi\right| d \sigma= & \lim _{n \rightarrow \infty} \int_{\Gamma}\left|\gamma\left(v_{1}^{n}\right) \Phi\right| d \sigma \\
& \leq \lim _{n \rightarrow \infty}\left(\iint_{\Gamma}\left|\gamma\left(v_{1}^{n}\right)\right|^{2} d \sigma\right)^{1 / 2}\left(\int_{\Gamma}|\varphi|^{2} d \sigma\right)^{1 / 2},
\end{aligned}
$$

$$
\lim _{n \rightarrow \infty}\left\|\gamma\left(v_{1}^{n}\right)\right\|_{L^{2}(\Gamma)}\|\varphi\|_{L^{2}(\Gamma)} \leq 1 \quad \text { for }\left(v_{1}^{n}\right) \in K
$$

From which we deduce that $\left\|\gamma\left(v_{1}\right)\right\|_{2}^{2}(\Gamma) \leq 1$.

Also, to show that $K_{1}$ is convex, let $v_{1}$ and $v_{2}$ be two elements in $K_{1}$, i.e.

$$
\left\|\gamma\left(v_{1}\right)\right\|_{l^{2},(r)} \leq 1 \text { and }\left\|\gamma\left(v_{2}\right)\right\|_{l_{1}^{2}(\Gamma)} \leq 1 .
$$

Let $\lambda$ be a number such that $0 \leq \lambda \leq 1$. Now $\lambda v_{1}+(1-\lambda) v_{2} \in K_{1} ; \gamma$ is a continuous linear map, therefore

$$
\begin{aligned}
\left\|\gamma\left(\lambda v_{1}+(1-\lambda) v_{2}\right)\right\|_{L^{2}(\Gamma)} & \leq\left\|\lambda \gamma\left(v_{1}\right)+(1-\lambda) \gamma\left(v_{2}\right)\right\|_{L^{2}(I)} \leq\left\|\lambda \gamma\left(v_{1}\right)\right\|_{L^{2}(\Gamma)}+\left\|(1-\lambda) \gamma\left(v_{2}\right)\right\|_{L^{2}(\Gamma)} \\
& \leq \lambda\left\|\gamma\left(v_{1}\right)\right\|_{L^{2}(\Gamma)}+(1-\lambda)\left\|\gamma\left(v_{2}\right)\right\|_{L^{2}(\Gamma)} \leq \lambda+(1-\lambda)=1 .
\end{aligned}
$$

Now consider $f \in L^{2}(\Gamma)$, then the problem of minimizing $J$ where

$$
J\left(v_{1}, v_{2}\right)=\left(\left(\frac{1}{2}\left(v_{1}, v_{1}\right)_{v_{1}},-\cdot\left(f, v_{1}\right)_{v_{1}}\right), v_{2}\right)
$$

on the closed convex set $K_{1}$ is equivalent to finding $u \in K_{1}$ satisfying the inequality

where $\left(f, v_{1}-u\right)_{L^{2}(r)}=1,\left(v_{1}-u\right)$

$$
\begin{aligned}
\left(a\left(u, v_{1}-u\right), v_{2}\right) \equiv & \left(\left(u, v_{1}-u\right)_{v_{1}}, v_{2}\right) \\
& \geq\left(\left(f, v_{1}-u\right)_{L^{2}(r)}, v_{2}\right) \quad \forall v_{1} \in K_{1}
\end{aligned}
$$


Therefore, assuming the solution $u$ ( which exists and unique by Theorem 3 ) is sufmciently regular, we can interpret $u$ as follows:

By Green's formula (1), we have

$$
\int_{0}(-\Delta u+u-f)\left(v_{1}-u\right) d x+\int_{I} \frac{\partial \underline{u}}{\partial \underline{n}}\left(v_{1}-u\right) d \sigma \geq 0, \quad \forall v_{1} \in K_{1} .
$$

$\Omega, \Gamma$ are subsets of $\mathbf{R}^{\mathbf{m}}$.

If $\varphi \in O(\Omega)$ then the houndary integral vanishes for $v_{1}=u \pm \varphi$ which belongs to $K_{1}$ and

$$
\int_{\Omega}(-\Delta \mathbf{u}+\mathbf{u}-\mathbf{f}) \varphi d x \geq 0
$$

which implies that $-\Delta \mathbf{u}+\mathbf{u}-\mathbf{f} \geq \mathbf{0}$ in $\Omega$.

Next since $v_{1}=2 u$ and $v_{1}=\frac{1}{2} u$ are both belong to $K_{1}$, we have

$$
\int_{\Gamma} \frac{\partial \underline{\mathbf{u}}}{\partial \underline{\mathbf{n}}} \mathbf{u d \sigma}=\mathbf{0}
$$

which implies that $\frac{\partial u}{\partial \underline{n}} u=0$ on $\Gamma$.

Thus the minimal of $J$ is equivalent to the solution of the following problem

$$
\left\{\begin{array}{lc}
-\Delta \mathbf{u}+\mathbf{u}-\mathbf{r} \geq 0 & \text { in } \Omega \\
\frac{\partial \mathbf{u}}{\partial \underline{\mathbf{n}}} \mathbf{u}=\mathbf{0} & \text { on } \Gamma \\
\frac{\partial \mathrm{u}}{\partial \underline{\mathbf{n}}} \mathbf{u} \geq 0 & \text { on } \Gamma \\
\mathbf{u} \geq \mathbf{0} & \text { on } \Gamma
\end{array}\right.
$$

One can also deduce from (I) that on the subset of $I$ where $u>0$, $u$ satisfies the homogeneous Neumann condition $\frac{\partial \underline{u}}{\partial \underline{n}}=0$.

From the Corollary 4 , and since $K_{2}$ is a nonempty closed convex set in $v_{2}$, we then have

$$
J\left(v_{1}, u_{2}\right)= \begin{cases}\max _{v_{2}}\left\{J\left(v_{1}, v_{2}\right) ; v_{2} \in K_{2}\right\} & \text { if } v_{1} \in K_{1}, \\ +\infty & \text { if } v_{1} \notin K_{1}\end{cases}
$$

i.e.,

$$
J\left(v_{1}, u_{2}\right)= \begin{cases}\max _{v_{2}}\left\{\left(\left(\frac{1}{2} a\left(v_{1}, v_{1}\right)-L\left(v_{1}\right)\right), v_{2}\right) ; v_{2} \in K_{2}\right\} & \text { if } v_{1} \in K_{1}, \\ +\infty & \text { if } v_{1} \notin K_{1}\end{cases}
$$

7. THEOREM. Suppose the functional

$$
J: H^{1}(\Omega) \rightarrow R
$$

is given by

$$
J(v)=\frac{1}{2} a(v, v)-I(v)
$$

and the closed convex set $K$ of $V=H^{1}(\Omega)$ is given by

$$
K:=\left\{v ; v \in H^{1}(\Omega), \quad 1-\operatorname{grad}^{2} v(x) \geq 0 \text { a.e. in } \Omega\right\}
$$

where

$$
1-\operatorname{grad}^{2} u(x) \in L^{1}(\Omega) \quad \text { and }\left(L^{\prime}(\Omega)\right)^{\prime}=L^{\infty}(\Omega) .
$$

Then the lagrangian

$$
\mathcal{L}(v, \mu)=J(v)+\left\langle\mu, \quad v>L^{\infty}(0) \times L^{1}(0)\right.
$$


associated to the primal problem (finding $u \in K$ such that $J(u) \leq J(v), \forall \quad v \in K$ ), has a minimax point ( saddle point).

PROOF. Let $\ell>0$ be any real number. We consider the subsets $K_{\ell}$ and $\Lambda_{\ell}$ of $H^{1}(\Omega)$ and $\Lambda$ respectively are defined by

$$
\begin{aligned}
& \mathrm{K}_{\ell}=\left\{v ; v \in \mathrm{H}^{1}(\Omega), 0 \leq 1-\operatorname{grad}^{2} \mathrm{v}(\mathrm{x}) \leq \ell \text { a.e. in } \Omega\right\} \\
& \Lambda_{\ell}=\left\{\mu ; \mu \in \mathrm{L}^{\infty}(\Omega), \quad 0 \leq \mu \leq \ell \text { in } \Omega\right\}
\end{aligned}
$$

such that $\Lambda_{\ell}$ being the cone in infinite dimensional Banach space.

Frist, we show that $K_{\ell}$ and $\Lambda_{\ell}$ are convex sets in $\Omega$.

Let $v_{1}, v_{2} \in K_{\ell}$ i.e.,

$$
\int_{\Omega}\left|1-\operatorname{grad}^{2} v_{1}(x)\right| d x \leq \ell^{1 / 2} \text { a.e. in } \Omega,
$$

and

$$
\int_{0}\left|1-\operatorname{grad}^{2} v_{2}(x)\right| d x \leq \ell^{1 / 2} \quad \text { a.e. in } \Omega .
$$

Let $0 \leq \lambda \leq 1$ we have

$$
\begin{aligned}
\int_{0}\left|1-\operatorname{grad}^{2}\left(\lambda v_{1}(x)+(1-\lambda) v_{2}(x)\right)\right| d x & =\int_{0}\left|1-\lambda \operatorname{grad}^{2} v_{1}(x)-(1-\lambda) \operatorname{grad}^{2} v_{2}(x)\right| d x \\
& =\int_{0}\left|\lambda\left(1-\operatorname{grad}^{2} v_{1}(x)\right)+(1-\lambda)\left(1-\operatorname{grad}^{2} v_{2}(x)\right)\right| d x \\
& \leq \int_{\Omega}\left|\lambda\left(1-\operatorname{grad}^{2} v_{1}(x)\right)\right| d x+\int_{0}\left|(1-\lambda)\left(1-\operatorname{grad}^{2} v_{2}(x)\right)\right| d x \\
& \leq \lambda \sqrt{\ell}+(1-\lambda) \sqrt{\ell}=\sqrt{\ell} \quad \text { a.e. in } \Omega .
\end{aligned}
$$

and hence is a convex set.

Now let $\mu_{1}, \mu_{2} \in \Lambda_{\ell}$ i.e., $\operatorname{Sup}\left|\mu_{1}\right| \leq \ell$ in $\Omega$ and $\operatorname{Sup}\left|\mu_{2}\right| \leq \ell$ in $\Omega$, for $0 \leq \lambda \leq 1$ we get

$$
\begin{aligned}
\operatorname{Sup}\left|\lambda \mu_{1}+(1-\lambda) \mu_{2}\right| \leq \operatorname{Sup}\left(\left|\lambda \mu_{1}\right|+\left|(1-\lambda) \mu_{2}\right|\right) & \text { in } \Omega \\
& \leq \operatorname{Sup}\left|\lambda \mu_{1}\right|+\operatorname{Sup}\left|(1-\lambda) \mu_{2}\right| \leq \lambda \ell+(1-\lambda) \ell=\ell \text { in } \Omega
\end{aligned}
$$

Thus $\Lambda_{\ell}$ is a convex set in $\Omega$. And $\Lambda_{\ell}$ is compact in the dual weak topology of $L^{\infty}(\Omega)$. Since $K_{\ell}$ is a closed bounded set in the Hilbert space $H^{1}(\Omega), K_{\ell}$ is weakly compact. We consider $H^{\prime}(\Omega)$ with its weak topology.

Now $H^{1}(\Omega)=V$ with the weak topology is a Hausdorf topological vector space.

All the hypothesis of the theorem of Ky Fan and Sion are satisfied by $\mathrm{K}_{\ell}, \Lambda_{\ell}$ and $\mathcal{L}$ in view of (I) and (ii). Hence

$$
\stackrel{L}{:} \mathbf{K}_{\ell} \times \Lambda_{\ell} \rightarrow \mathbf{R}
$$

has a saddle point $\left(u_{\ell}, \lambda_{\ell}\right)$, i.e., there exist $\left(u_{\ell}, \lambda_{\ell}\right) \in K_{\ell} \times \Lambda_{\ell}$ such that

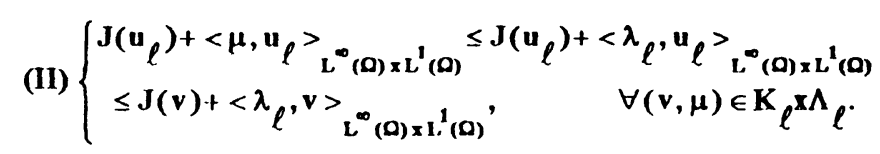


We shall show that if we choose $\ell>0$ sufmciently large then such a saddle point can be obtained independent of $\ell$.

For this we shall first prove $\left\|u_{\ell}\right\|_{Y^{1}(\Omega)}$ and $\lambda_{\ell}$ are bounded by constants independent of $\ell$. If we take $\mu=0 \in \Lambda_{\ell}$ in (II) we get

$$
\mathrm{J}\left(\mathbf{u}_{\ell}\right) \leq \mathrm{J}(\mathrm{v})+\left\langle\lambda_{\ell^{\prime}}, \mathrm{v}>_{\mathbf{L}^{\infty}(\Omega) \times \mathbf{L}^{\prime}(\Omega)}, \quad \forall \mathbf{v} \in \mathrm{K}_{\ell}\right.
$$

taking $v=0 \in K_{\ell} \cap K$ such that

$$
1-\operatorname{grad}^{2} v(x) \geq 0 \quad \text { a.e. in } \Omega
$$

we get

$$
J\left(u_{\ell}\right) \leq J(0) \text { for } 1-\operatorname{grad}^{2} v(x) \geq 0 \quad \text { a.e. in } \Omega
$$

On the other hand, since $a\left(u_{\ell}, u_{\ell}\right) \geq 0$ and since $u_{\ell} \in K_{\ell}$

$$
\mathrm{L}\left(\mathrm{u}_{\ell}\right) \leq\|\mathrm{L}\|_{v}\left\|1-\operatorname{grad}^{2} v(\mathrm{x})\right\|_{\mathrm{L}^{1}(\Omega)} \leq \ell\|\mathrm{L}\|_{v} \quad \text { a.e. in } \Omega
$$

and hence

$$
J\left(u_{\ell}\right)=\frac{1}{2} a\left(u_{\ell}, u_{\ell}\right)-L\left(u_{\ell}\right) \geq-\ell\|L\|_{\cdot} \quad \text { a.e. in } \Omega
$$

Thus we have

$$
-\ell\|\mathbf{L}\|_{v} \leq \mathrm{J}\left(\mathbf{u}_{\ell}\right) \leq \mathrm{J}(\mathbf{0}) \quad \text { a.e. in } \Omega
$$

Now by coercivity of a (., .) and (4) we find

$$
\alpha\left\|\mid u_{\ell}\right\| \|^{2} \leq a\left(u_{\ell}, u_{\ell}\right):=2\left(J\left(u_{\ell}\right)+L\left(u_{\ell}\right)\right) \leq 2\left(J(0)+\|L\|_{v}\left\|u_{\ell}\right\|\right) \text { a.e. in } \Omega
$$

with a constant $\alpha>0$ (independent of $\ell$ ) and $\|.1\|$ defined the norm $\|\cdot\|_{H}^{1}(0)$.

By using the inequality

$$
\|L\|_{v}\left\|\left|u_{\ell}\right|\right\| \leq \varepsilon\left\|\mid u_{\ell}\right\|\left\|^{2}+\frac{1}{\varepsilon}\right\| L \|_{v}^{2} . \quad \text { for any } \varepsilon>0
$$

with $\varepsilon=\alpha / 4>0$, we optain

$$
\alpha\left\|\left|u_{\ell}\right|\right\| \leq 2 J(0)+\frac{\alpha}{2}\left\|\left|u_{\ell}\right|\right\|^{2}+\frac{8}{\alpha}\|L\|_{v^{\prime}}^{2}
$$

Therefore,

$$
\left\|u_{\ell}\right\| \|^{2} \leq \frac{4}{\alpha}\left(J(0)+\frac{4}{\alpha}\|L\|_{v^{\prime}}^{2}\right)
$$

This proves that there exists a constant $C_{1}>0$ such that

$$
\left\|u_{\ell}\right\| \|^{2} \leq C_{1} \quad \forall \ell, \quad \text { a.e. i } \Omega \text {. }
$$

We observe that since $J$ satisfies all assumptions of Theorem (3), there will exists a unique global minimum in $V=H^{1}(\Omega)$ i.e., there exists a unique $\tilde{u} \in H^{1}(\Omega)$ such that

$$
\mathrm{J}(\tilde{\mathrm{U}}) \leq \mathrm{J}(\mathbf{v}), \quad \forall \mathbf{v} \in \mathrm{V}
$$

But, if we take $v=1 \in K_{\ell}$ in the inequality (3) we get

$$
J\left(u_{\ell}\right)+\sup \lambda_{\ell} \leq J(1) \text {. }
$$

The last two inequalities imply that

$$
\mathrm{C}_{2}=-\mathrm{J}(\tilde{\mathbf{n}})+\mathrm{J}(1) \geq \sup \lambda_{\ell} \geq 0
$$

which proves that $\lambda_{\ell}$ is also bounded, $\ell$ may be taken as follows

$$
\ell>\max \left(C_{1}, 2 C_{2}\right)>0
$$

Next we show that (II) holds for any $\mu \in \Lambda$ For this we write the frist inequality of (II) in the form 
which implies that

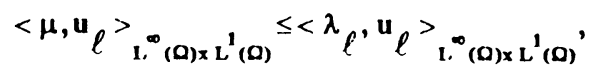

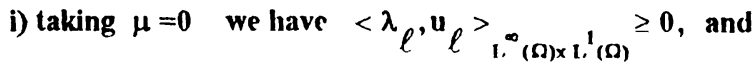

ii) taking $\mu=2 \lambda_{\ell} \leq 2 C_{2}<\ell$ we have $<\lambda_{\ell}, u_{\ell}>_{1,(0) \times \mathrm{L}^{1}(\Omega)} \leq 0$.

Thus

$$
\left\langle\lambda _ { \ell } , \mathrm { u } _ { \ell } > _ { \mathrm { L } ^ { \infty } ( \Omega ) \times \mathrm { L } ^ { \prime } ( \Omega ) } = 0 \text { and } \left\langle\mu, \mathrm{u}_{\ell^{\prime}}>_{\mathrm{L}^{\infty}(\Omega) \times \mathrm{L}^{\prime}(\Omega)} \leq 0, \quad \forall \mu \in \Lambda_{\ell}\right.\right.
$$

In particular, $\mu=\ell \in \Lambda_{\ell}$ and so $\left\langle\ell, \|_{\ell^{\prime}}>_{L^{\infty}(\Omega) \times L^{\prime}(\Omega)} \leq 0\right.$ which means that

$$
<\mu, \mathrm{u}_{\ell^{\infty}}>_{\mathrm{L}_{(\Omega) \times \mathrm{L}^{1}(0)}} \leq 0 \text { for any } \mu \geq 0 \text {, }
$$

and therefore

$$
\mathcal{L}\left(\mathbf{u}_{\ell}, \mu\right) \leq \mathcal{L}\left(\mathbf{u}_{\ell}, \lambda_{\ell}\right) \leq \mathcal{L}\left(\mathbf{v}, \lambda_{\ell}\right) \quad \forall \mu \geq 0 \text { and } \mathbf{v} \in \mathbf{K}_{\ell}
$$

where $\ell>\max \left(C_{1}, 2 C_{2}\right)$.

We have now only to show that the inequality (9) holds for any $v \in \mathrm{H}^{1}(\Omega)=\mathrm{V}$.

For this, we note that $\left\|u_{\ell}\right\| \|^{2} \leq C_{1}<\ell$ a.e. in $\Omega$, and hence we can find an $r>0$ such that the ball

$$
B\left(u_{\ell}, r\right)=\left\{v ; v \in H^{1}(\Omega): 1-\operatorname{grad}^{2}\left(v(x)-u_{\ell}(x)\right)<r \text { a.e. in } \Omega\right\}
$$

is contained in the ball

$$
B\left(u_{\ell}, r\right)=\left\{v ; v \in H^{1}(\Omega): 1-\operatorname{grad}^{2} v(x)<\ell \text { a.e. in } \Omega\right\}
$$

In fact, it is enough to take $0<r<\left(\ell-C_{1}\right) / 2$. Now the functional

$$
\begin{aligned}
& \mathcal{L}\left(, \lambda_{\ell}\right): v \rightarrow \mathcal{L}\left(v, \lambda_{\ell}\right) \\
& \mathcal{L}\left(v, \lambda_{\ell}\right)=J(v)+<\lambda_{\ell}, v>_{\mathrm{L}^{\infty}(\Omega) \times \mathrm{L}^{1}(0)}
\end{aligned}
$$

has a local minimum in $B\left(u_{\ell}, r\right)$. But since this functional is convex such a minimum is also a global minimum. This means that

$$
\inf _{v \in \mathbb{B}\left(\mathrm{u}_{\ell}, \mathrm{r}\right)} \mathcal{L}\left(\mathrm{v}, \lambda_{\ell}\right)=\inf _{\mathrm{v} \in \mathrm{V}} \mathcal{L}\left(\mathrm{v}, \lambda_{\ell}\right)
$$

On the other hand, since $B\left(u_{\ell}, r\right) \subset K_{\ell}$, we see from (9) that

$$
\mathscr{L}^{\prime}\left(u_{\ell}, \mu\right) \leq \mathcal{L}\left(u_{\ell}, \lambda_{\ell}\right) \leq \inf _{v \in K_{\ell}} \mathcal{L}\left(v, \lambda_{\ell}\right) \leq \inf _{v \in B\left(u_{\ell}, r\right)} \mathcal{L}\left(v, \lambda_{\ell}\right)=\inf _{v \in V} \mathscr{L}\left(v, \lambda_{\ell}\right) .
$$

In other words, we have

$$
\mathcal{L}\left(u_{\ell}, \mu\right) \leq \mathcal{L}\left(u_{\ell}, \lambda_{\ell}\right) \leq \mathcal{L}\left(v, \lambda_{\ell}\right), \quad \forall v \in v \text {, and } \forall \mu \geq 0,
$$

which means that $\mathcal{C}$ has a saddle point.

\section{REFERENCES}

1. JEAN CEA, Lectures on Optimizing-Theory and Algorithms, Narosa Publishing House, New Delhi, 1978

2. Rockafellar, R.T., Convex Analysis, Princeton, New Jersy, 1970.

3. Vladimirov, V. S. Equations of Mathematical Physics, Mir Publishers, Moscow, 1984. 


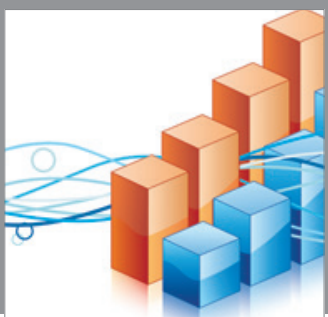

Advances in

Operations Research

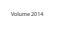

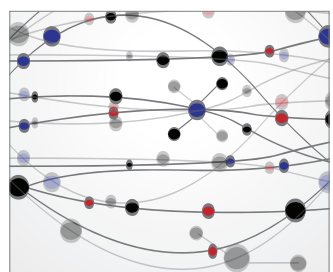

\section{The Scientific} World Journal
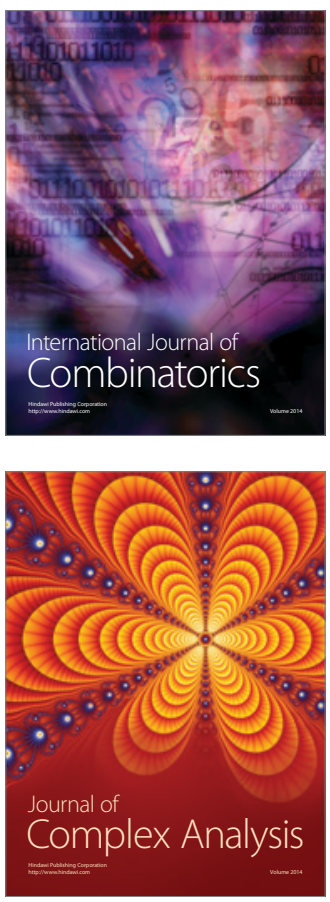

International Journal of

Mathematics and

Mathematical

Sciences
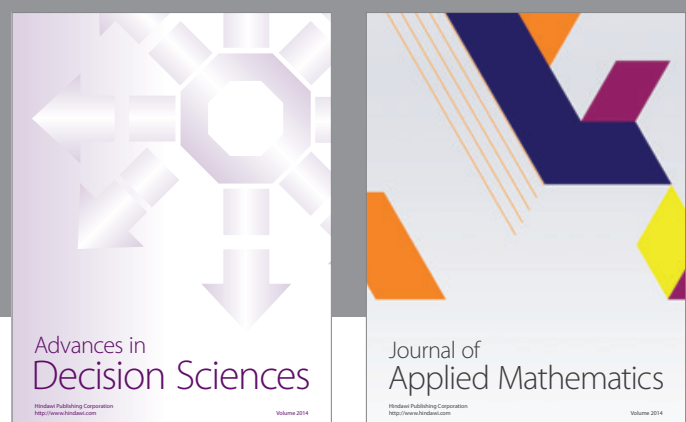

Journal of

Applied Mathematics
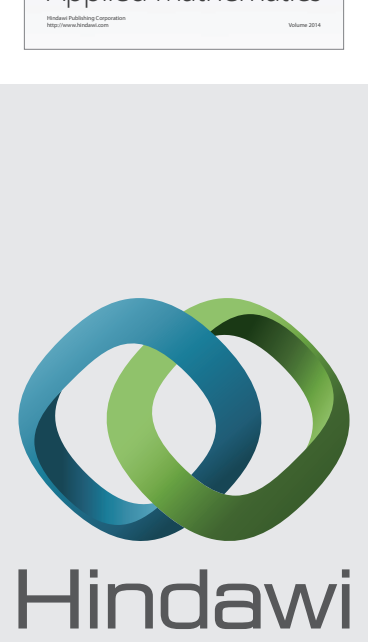

Submit your manuscripts at http://www.hindawi.com
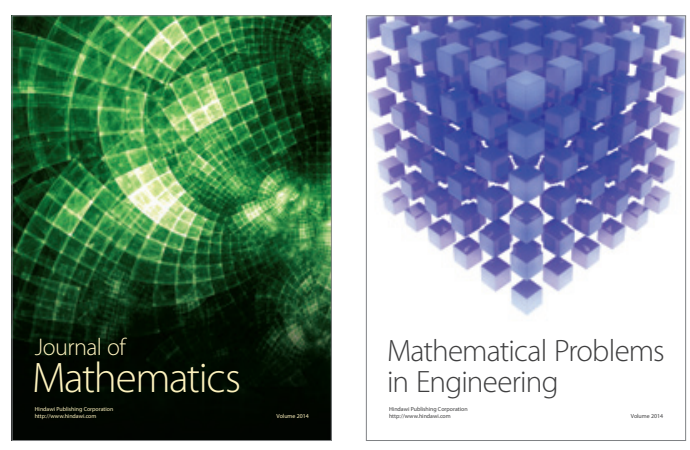

Mathematical Problems in Engineering
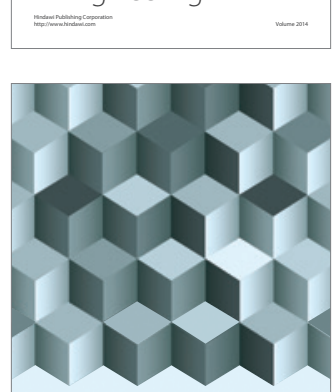

Journal of

Function Spaces
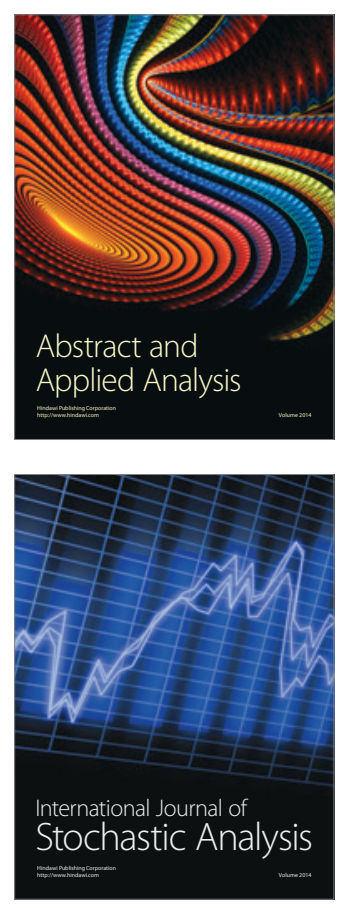

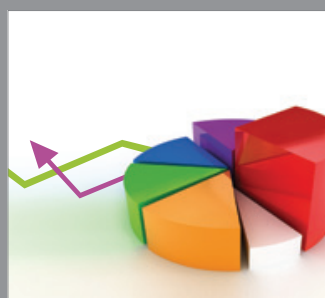

ournal of

Probability and Statistics

Promensencen
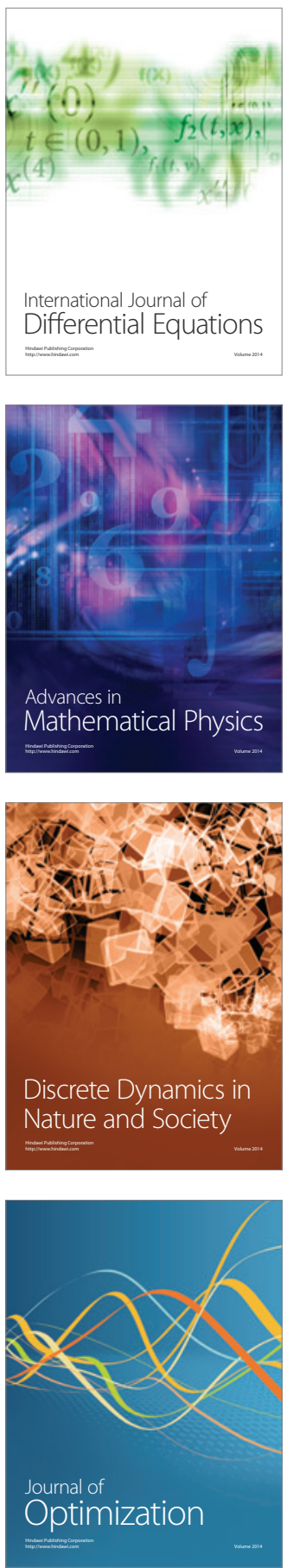